\title{
Zygomycotic necrotizing fasciitis in immunocompetent patients: a series of 18 cases
}

\author{
Deepali Jain ${ }^{1}$, Yashwant Kumar ${ }^{1}$, Rakesh K Vasishta ${ }^{1}$, Logasundaram Rajesh ${ }^{1}$, \\ Sanjib K Pattari ${ }^{1}$ and Arunaloke Chakrabarti ${ }^{2}$ \\ ${ }^{1}$ Department of Histopathology, Post-graduate Institute of Medical Education and Research, Chandigarh, \\ India and ${ }^{2}$ Department of Microbiology, Post-graduate Institute of Medical Education and Research, \\ Chandigarh, India
}

\begin{abstract}
Necrotizing fasciitis is most often associated with bacterial infections. Zygomycosis is an uncommon infection causing necrotizing fasciitis. We report 18 such cases of zygomycotic necrotizing fasciitis, of these, 15 were immunocompetent. Of the eight cases cultured, five were positive for Apophysomyces elegans. A retrospective case review conducted at a tertiary referral center, from 1998 to 2004, 18 cases of fungal necrotizing fasciitis were diagnosed based on histomorphology of fungal organisms; and in few of the cases diagnosis was supported by mycologic culture reports. Of the total of 18 cases, culture report was available in eight cases, and out of which five of them grew $A$. elegans. Fifteen patients were immunocompetent. Clinical presentation, mycologic findings and histopathologic results were evaluated. A review of the literature pertaining to $A$. elegans infection was also done. Histopathologic examination showed broad, predominantly aseptate and occasional pauciseptate, thin-walled fungal hyphae with occasional angioinvasion. To the best of our knowledge, this is the first largest series of zygomycotic necrotizing fasciitis from India. Herein, we present data on 18 cases of necrotizing fasciitis assosiated with zygomycosis. Most of the cases in our series were immunocompetent. Nonsuppurative necrosis with presence of typical fungal profiles was important histologic feature. Zygomycosis must be considered in the differential diagnosis not only in immunocompromised patients but also in the absence of any underlying disorders.
\end{abstract}

Modern Pathology (2006) 19, 1221-1226. doi:10.1038/modpathol.3800639; published online 2 June 2006

Keywords: necrotizing fasciitis; fungal; zygomycosis; Apophysomyces elegans; immunocompetent

Necrotizing fasciitis is a rare and often fatal softtissue infection. It typically begins with trauma; although the inciting event may be as seemingly innocuous as a simple contusion, minor burn, or insect bite. The disease occurs more frequently in diabetics, alcoholics, immunosuppressed patients, i.v. drug users, and patients with peripheral vascular disease. ${ }^{1}$ Although it can occur in any region of the body, the abdominal wall, perineum and extremities are the most common sites of infection. ${ }^{2}$ Clinical presentation involves fever, cellulitis, edema, crepitus, bullae, necrosis and sepsis. Operative findings include fascial and subcutaneous tissue necrosis with or without myonerosis. ${ }^{3}$ Necrotizing fasciitis may occur as a consequence of infection with

Correspondence: Dr D Jain, MD, DNB, Department of Pathology, All India Institute of Medical Sciences, New Delhi, 110029, India. E-mail: deepalipgi@rediffmail.com

Received 20 March 2006; revised 27 April 2006; accepted 28 April 2006; published online 2 June 2006
Streptococcus pyogenes or as a result of a polymicrobial synergistic infection caused by aerobic, anaerobic, Gram-positive and Gram-negative organisms. ${ }^{4,5}$ Fungal infection although reported, is not a common finding in necrotizing fasciitis; only a handful of case reports are available in literature. ${ }^{6,7}$ In this study, we described a large series of fungal necrotizing fasciitis cases due to zygomycosis with their clinical, histopathologic and microbiologic findings. With the best of our knowledge this is the largest series of fungal necrotizing fasciitis from India.

\section{Materials and methods}

Cases of necrotizing fasciitis were analyzed over a period of 7 years (1998-2004) from the archives of Department of Histopathology, Post graduate institute of medical education and research, Chandigarh, India. A total of 58 cases of necrotizing 
Table 1 Clinical, microbiological and histopathologic data of 18 patients

\begin{tabular}{|c|c|c|c|c|c|c|c|c|}
\hline \multirow[t]{3}{*}{$S N$} & \multirow[t]{3}{*}{ Age/sex } & \multirow[t]{3}{*}{ Site/surgical procedure } & \multirow[t]{3}{*}{ Clinical details } & \multicolumn{4}{|c|}{ Microbiology } & \multirow[t]{3}{*}{ Remarks } \\
\hline & & & & \multirow[b]{2}{*}{$\begin{array}{c}\text { Pus cells } \\
\text { (neutrophills) }\end{array}$} & \multicolumn{2}{|c|}{ Smear report } & \multirow[t]{2}{*}{ Culture } & \\
\hline & & & & & Hyphae & Bact & & \\
\hline 1 & $50 \mathrm{~F}$ & Lt Gluteal D & IM inj followed by abscess & + & Aseptate & - & No growth & \\
\hline 2 & $48 \mathrm{M}$ & Lt Gluteal D & Stab injury & & & & NP & \\
\hline 3 & $72 \mathrm{M}$ & Rt interdigital cleft D & $\begin{array}{l}\text { Case of adenoca caecum, Rt } \\
\text { hemicolectomy done }\end{array}$ & & & & $\mathrm{NP}$ & Immunosuppressed \\
\hline 4 & $20 \mathrm{M}$ & Lt Thigh D & RSA, motor vehicle & - & Septate & - & A. flavum & Coexistent Aspergillus \\
\hline 5 & $22 \mathrm{M}$ & Abdominal wall D & RSA, motor vehicle & ++ & - & + & No growth & \\
\hline 6 & $43 \mathrm{~F}$ & Ant abdominal wall D & DM on insulin, asthmatic on prednisolone & & & & $\mathrm{NP}$ & $\begin{array}{l}\text { Immunosuppressed, } \\
\text { coexistent Candida }\end{array}$ \\
\hline 7 & $45 \mathrm{M}$ & Flank Lt D & Splenic abscess and flank cellulitis & + & Aseptate & - & A. elegans & \\
\hline 8 & $55 \mathrm{~F}$ & Rt loin D & Perinephric abscess & + & Aseptate & - & A. elegans & \\
\hline 9 & $60 \mathrm{~F}$ & Rt thigh \& leg amputation & \# SOF compound Grade III & + & Aseptate & + & A. elegans & \\
\hline 10 & $30 \mathrm{~F}$ & Chest wall D & RSA, motor vehicle & + & Aseptate & + & A. elegans & \\
\hline 11 & $65 \mathrm{M}$ & Lt gluteal D & IM inj followed by abscess & & & & NP & \\
\hline 12 & $20 \mathrm{M}$ & Chest wall and abdomen D & Fall from height & & & & NP & \\
\hline 13 & $60 \mathrm{M}$ & Rt leg D & Deep Burn & + & Aseptate & - & A. elegans & \\
\hline 14 & $20 \mathrm{M}$ & Chest wall D & Bullet injury & & & & $\mathrm{NP}$ & \\
\hline 15 & $55 \mathrm{M}$ & Rt gluteal D & Gluteal abscess & & & & NP & \\
\hline 16 & $35 \mathrm{M}$ & Rt gluteal D & $\begin{array}{l}\text { Invasive gluteal mucormycosis with } \\
\text { extensive necrosis of soft tissue }\end{array}$ & & & & $\mathrm{NP}$ & \\
\hline 17 & $22 \mathrm{M}$ & $\begin{array}{l}\text { Lt leg, disarticulation of gangrenous } \\
\text { limb }\end{array}$ & RSA, motor vehicle & & & & $\mathrm{NP}$ & \\
\hline 18 & $5 \mathrm{M}$ & Multiple subcut nodules & ALL-L1 & & & & NP & Immunosuppressed \\
\hline
\end{tabular}

Abbreviations: Adenoca, adenocarcinoma; A. elegans, Apophysomyces elegans; A. flavum, Aspergillus flavum; ALL, acute lymphoblastic leukemia; Bact, Bacteria; Crypto, Cryptococcus; D, debridement; DM, diabetes mellitus; F, female; \#, fracture; IM inj, intramuscular injection; Lt, left; M, male; NP, not performed; Rt, right; RSA, road-side accident; SN, serial number; SOF, shaft of femur; Subcut, subcutaneous. 
fasciitis were diagnosed. While searching for etiologic factors of necrotizing fasciitis, through SNOMED coding system, 18 cases of fungal necrotizing fasciitis were found from the database. Diagnosis was confirmed by combination of clinical and histomorphologic findings. Moreover in eight patients it was well supported by mycologic culture report.

\section{Microbiologic Examination}

Debrided tissues were inoculated onto blood agar, inhibitory mold agar and malt extract agar. The organism was subcultured to potato dextrose agar, and after 3 days of incubation at $30^{\circ} \mathrm{C}$, a block of the medium was placed which was then incubated at $37^{\circ} \mathrm{C}$.

\section{Histopathologic Examination}

Resected tissue from all cases was fixed in $10 \%$ neutral-buffered formalin, routinely processed and paraffin embedded. Sections (5 $\mu \mathrm{m}$ thick) were stained by hematoxylin and eosin (H\&E) stain. Three histopathologists independently reviewed the H\&E slides along with relevant histochemical stains and reconfirmed the original histopathologic diagnoses. Periodic acid Schiff (PAS) and Silver methenamine stain for fungus were performed in all cases. Neither immunohistochemistry nor molecular techniques were carried out for diagnosis of these cases.

\section{Results}

A total of 18 patients of fungal necrotizing fasciitis were analyzed at this tertiary care center, during a study period of 7 years, between January 1998 and December 2004.

\section{Clinical Profile}

The age ranged from 5 to 72 years (mean 40.3 years). There was male preponderance (13 cases) with male to female ratio of $2.6: 1$ (Table 1 ).

Of the total of 18 cases, three patients were immunosuppressed. Of these three, one patient was diabetic and the other two had history of malignancy and were receiving chemotherapy. In remaining 15 patients, no history of underlying immunosuppression was present. Four of the patients had history of motor vehicle road-side accident. Some kind of injury was present in 10 other cases. One patient had fracture neck of the femur. Lower extremities and gluteal region were involved in six and four cases, respectively. Eight cases presented with abdomen and chest wall involvement.

\section{Microbiologic Analysis}

Microbiologic data was available in eight patients (Table 1). Mycologic culture was not done in remaining patients as they were put on broad spectrum antibiotics, based on clinical diagnosis of polymicrobial necrotizing fasciitis. Macroscopically colonies were fluffy and cottony. Surface of the colony was initially white and turned brownish gray after some time. Sporangiophores with apophyses, and pyriform sporangia were identified. Smear examination revealed aseptate, broad $(4-8 \mu \mathrm{m}$ in diameter) and branching hyphae in six cases. Whereas one case showed septate hyphae on smear examination and grown as Aspergillus flavum on mycologic culture. Although on smear, bacteria were positive in three cases, bacterial cultures were sterile.

\section{Histological Examination}

Morphologically all cases exhibited predominantly aseptate and occasional sparsely septate, broad and branching hyphae, consistent with the morphology of zygomyces species (Figure 1). The angle of hyphal branching varied from $45^{\circ}$ to $90^{\circ}$. At places the hyphae had a compressed distorted appearance owing to the thin hyphal walls (Figure 2). In addition to zygomycosis, one case showed septate acute angle branching hyphae of Aspergillus invaded only the superficial necrotic tissue; and one other case revealed yeast forms of Candida. Necrotizing inflammation involving skin, subcutaneous tissue and deeper tissue including fat, underlying fascial layers and muscle was seen in all of the cases. Four cases showed necrosis of skeletal muscle. Focal or diffuse nonsuppurative necrosis of subcutaneous tissue was noticed in all cases (Figure 3). Most areas of the tissue showed coagulative necrosis with focal suppurative inflammation.

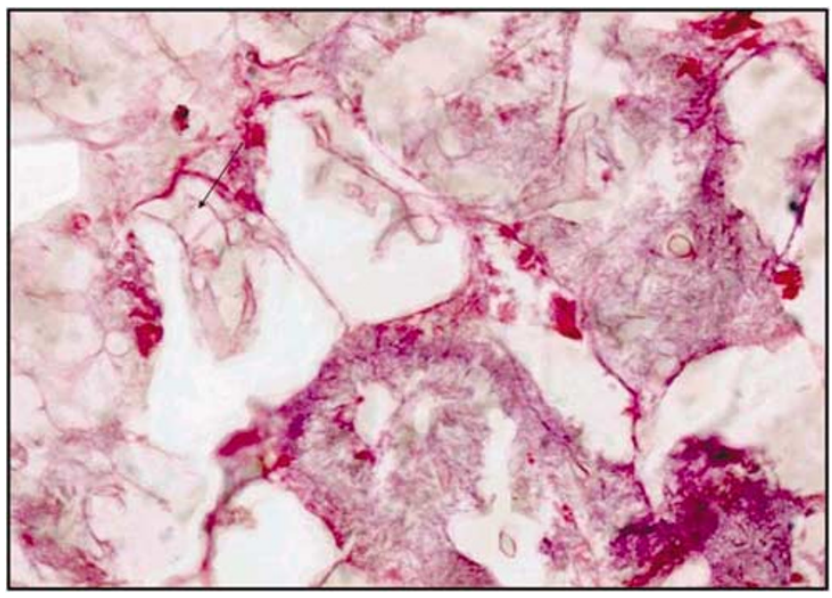

Figure 1 Acutely-to-obtusely branching, pauciseptate hyphae of zygomyces are present. The arrow designates a septum (H\&E, $\times 200)$. 


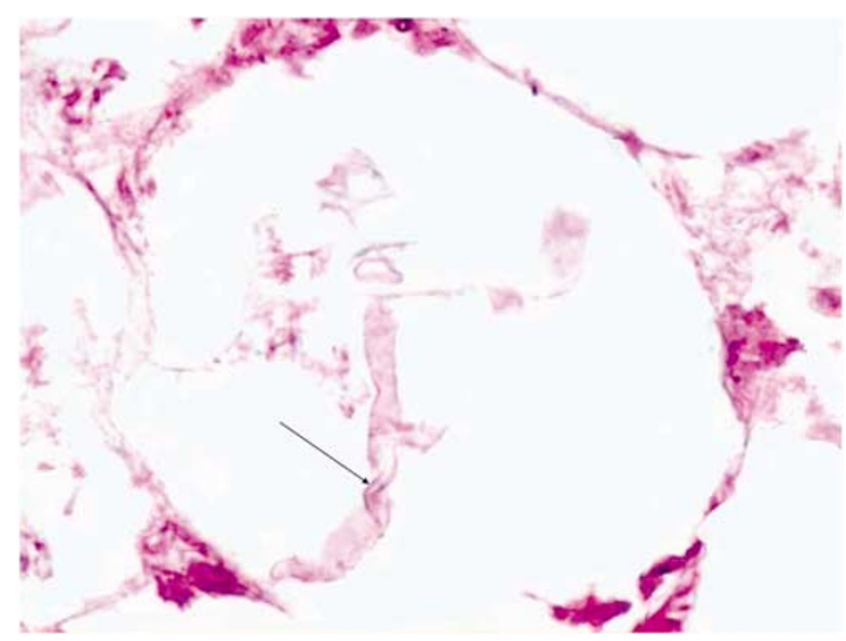

Figure 2 Broad aseptate thin walled fungal hyphae with collapse of their walls (arrow) (PAS, $\times 400)$.

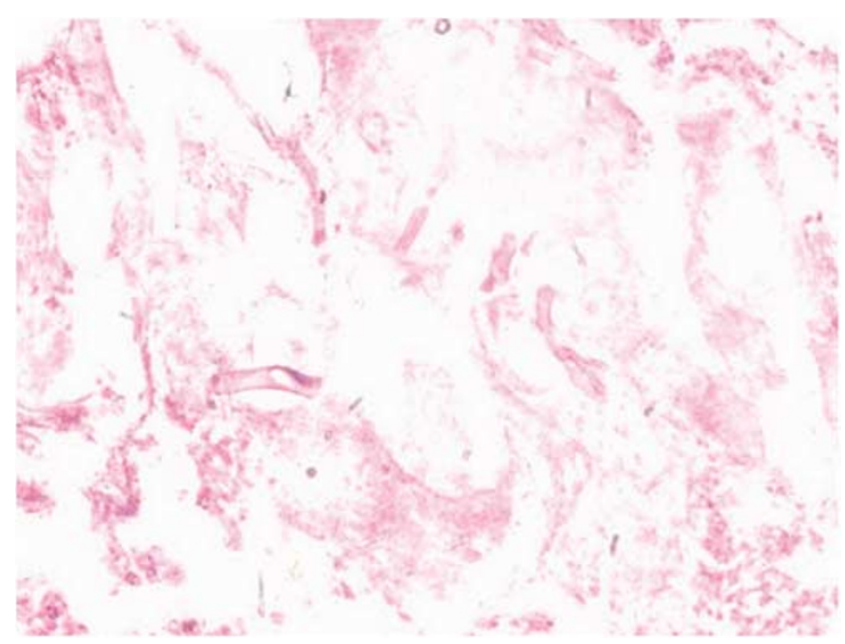

Figure 3 Photomicrograph shows subcutaneous non-suppurative necrosis with few fungal profiles $(\mathrm{H} \& \mathrm{E}, \times 200)$.

The fungal profiles were more numerous in the area, where there was nonsuppurative necrosis, rather than in densely inflammed areas. Vascular invasion was noticed in three (16\%) cases. Based on overall morphologic findings and in few cases with support of positive fungal culture, a diagnosis of zygomycotic necrotizing fasciitis was made.

\section{Discussion}

Necrotizing fasciitis is being attributed to a large variety of organisms, besides the originally described beta-hemolytic Streptococcus. Detailed microbiologic study of these infections revealed that these infections are polymicrobial; and fungal species are responsible for occasional cases. ${ }^{4-7}$ However, in the present series, we have found zygomycosis in $31.03 \%$ cases of necrotizing fasciitis, over a period of 7 years, which is significantly high. There are only few case reports available on fungal necrotizing fasciitis, however, information on a large series of patients with clinical, histological and microbiologic details is scant in the literature.

Zygomycetes class of fungi includes three orders that is Mucorales, Mortierellales and Entomophthorales. The majority of human illness is caused by the Mucorales. While disease is most commonly linked to Rhizopus spp., other organisms are also associated with human infection, including Mucor, Rhizomucor, Absidia, Apophysomyces, Saksenaea, Cunninghamella, Cokeromyces, and Syncephalastrum spp. Mortierellales, cause disease in animals, and Entomophthorales produce some relatively distinct syndromes and have dramatically different morphologies from Mucorales. ${ }^{8}$ Human zygomycosis caused by the Mucorales generally occurs in immunocompromised hosts as opportunistic infections. ${ }^{9}$ However, an increasing trend of mucormycosis in immunocompetent patients has been seen and reported recently by Sridhara et $a l^{10}$ from this institute. In which authors concluded that mucormycosis must be considered in the differential diagnosis not only in immunocompromised patients but also in the absence of any underlying disease. In immunocompromised patients, risk factors for development of zygomycosis include diabetes mellitus, neutropenia, sustained immunosuppressive therapy, chronic prednisone use, severe malnutrition and primary breakdown in the integrity of the cutaneous barrier such as trauma and surgical wounds. ${ }^{11,12}$ Histologically zygomycetes class of fungi exhibit broad, thin-walled, hyaline, often aseptate or pauciseptate hyphae with frequent angioinvasion. The hyphae of the zygomycetes appear thinner-walled compared to other fungal hyphae, which possibly accounts for their weaker staining with Gomori methenamine silver and PAS techniques relative to fungi with thicker cell walls. ${ }^{13}$ In contrast to the zygomycetes, Aspergillus tends to demonstrate an acute branching pattern and has narrower, more uniform septa. ${ }^{13}$ Vascular invasion that causes necrosis of the infected tissue, and perineural invasion are the most morbid features of these infections. ${ }^{8}$ The vasoinvasion was seen in only $16 \%$ of our cases. In contrast to findings of Frater et al, we did not find perineural invasion in the present series.

Generally, diagnosing mucormycosis almost always requires histopathologic evidence of fungal invasion of the tissues. Culturing organisms from an infected site is rarely sufficient to establish the diagnosis because the causative agent is ubiquitous, may colonize normal persons, and is a relatively frequent laboratory contaminant. In addition, the organism may be killed during tissue grinding, ${ }^{14}$ which is routinely used to process tissue specimens for culture. There are no reliable serologic, 
PCR-based or skin tests for mucormycosis. Therefore, the diagnosis should be made by biopsy of infected tissues. The genus and species of the infecting organism may be determined by culture of the infected tissue. However, the organism is rarely isolated from cultures of blood, cerebrospinal fluid, sputum, urine, feces or swabs of infected areas. ${ }^{15}$ As in our series, culture was available for only the minority of cases, hence the diagnosis of zygomycosis was based solely on histomorphology.

In the present series, most of the patients were immunocompetent (15/18), and had history of injury thus zygomycosis in these cases was attributed secondary to breakdown in the mucocutaneous barrier. Unfortunately microbiologic culture reports were available in only eight of these 15 patients. Of these eight, $A$. elegans was cultured in five $(62.5 \%)$ cases. Although in remaining three immunosuppressed patients (cases-3, 6 and 18) microbiologic data was not available, but zygomycotic necrotizing fasciitis in these cases could be attributed either to other members of zygomycetes family such as mucor or to Apophysomyces itself. As morphologically A. elegans and Mucor are similar and can not be distinguished. Moreover Apophysomyces was described in immunosuppressed patients rarely. ${ }^{8,16}$ Other members of Mucorales order besides A. elegans and Mucor are morphologically distinct, therefore might not be responsible pathogens in our cases. $^{8}$

A. elegans is a rare cause of human zygomycosis, and the infection is usually acquired via traumatic implantations such as burns or invasive procedures, in previously healthy patients. By far the most common site of disease manifestation in A. elegans infections is the cutaneous and subcutaneous tissue, with local invasion resulting into necrotizing fasciitis. ${ }^{17} \mathrm{~A}$. elegans is an uncommon human pathogen that was first isolated in 1979 from soil samples collected in an orchard in northern India. ${ }^{18}$ Kimura et $a l^{19}$ reviewed 16 cases of $A$. elegans. While reviewing the literature further, we found 13 additional case reports; and two large series from this institute (Table 2). The study from this institute by Chakrabarti et $a l^{29}$ highlighted the importance of increased awareness for early diagnosis of zygomycosis and aggressive management. The large number of cases in apparently healthy hosts and increased isolation of $A$. elegans in the same series were important characteristics of this disease in India.

Two patients in the present series revealed coexistence of Candida and Aspergillus (cases 4 and 6). One patient had evidence of a zygomycotic infection and Candida by light microscopy only, which was not confirmed by culture. A second patient's tissue grew $A$. flavum on culture but showed evidence of zygomyces by light microscopy only. Neither patient had both Candida and Aspergillus, in addition to a zygomycotic infection. Although immunosuppression is the major factor for Aspergillus infection but it may be local
Table 2 Literature review of $A$. elegans infection

\begin{tabular}{rll}
\hline SN & Studies & Site of lesion \\
\hline 1 & Chakrabarti et $a l^{20}$ & Craniofacial \\
2 & Caceres et $a l^{21}$ & Limb \\
3 & Brown et $a l^{22}$ & Rhinocerebral \\
4 & Burrell et $a l^{23}$ & Spine \\
5 & Fairley et $a l^{24}$ & Rhino-orbital-cerebral \\
6 & Garcia-Covarrubias et $a l^{25}$ & Rhino-orbital-cerebral \\
7 & Blair et $a l^{26}$ & Cutaneous \\
8 & Lesueur et $a l^{27}$ & Hand \\
9 & Wang et $a l^{28}$ & Cutaneous \\
10 & Chakrabarti et al $l^{29}$ (eight cases) & Cutaneous and \\
& & subcutaneous, renal, rhino- \\
& & orbital, disseminated \\
11 & Carter et $a l^{30}$ & Forearm \\
12 & Kordy et $a l^{31}$ & Deep soft tissue \\
13 & Ruiz et $a l^{32}$ & Lumbar region \\
14 & Andresen et $a l^{33}$ & Thigh \\
15 & Sridhara et $a l^{10}$ (three cases) & Rhino-orbital-cerebral \\
16 & Present series (five cases) & Thigh, chest, gluteal region \\
& & \\
\hline
\end{tabular}

colonizer or might have occurred due to nosocomial infection in this case. ${ }^{8}$

The present series describes large number of patients of zygomycotic necrotizing fasciitis with their clinical, histopathologic and microbiologic features. The most important histological finding in all the cases was nonsuppurative necrosis of subcutaneous fat. In fact, presence of nonsuppurative necrosis of subcutaneous fat without much inflammatory reaction should raise the suspicion for zygomycosis. Patients with necrotizing fasciitis had an overall mortality rate of $47.7 \%$. However, mortality in patients with superimposed zygomycosis rises to $80 \% .^{34}$ So an early diagnosis and management is important in this group. Unfortunately no follow-up information was available for the patients of present series.

To the best of our knowledge, this is the first largest series of zygomycotic necrotizing fasciitis cases from India. This study is to alert the pathologists that one should be cautious in diagnosing necrotizing fasciitis cases because all the time these cases may not be due to bacterial infection and may not be in immunocompromised patients. We report a series of 18 cases of fungal necrotizing fasciitis, eight with confirmatory cultures and the remainder with characteristic histomorphology. All the cases showed ribbon-like hyphae typical of the zygomycetes. Occasional septations, thin walls and broad-angle branching with angioinvasion are the few features, important for differentiation from other group of fungi.

\section{References}

1 Fontes Jr RA, Ogilvie CM, Miclau T. Necrotizing soft-tissue infections. J Am Acad Orthop Surg 2000;8: 151-158. 
2 Green RJ, Dafoe DC, Raffin TA. Necrotizing fasciitis. Chest 1996;110:219-229.

3 Pessa ME, Howard RJ. Necrotizing fasciitis. Surg Gynecol Obstet 1985;161:357-361.

4 Cherneski CL, Embil JM. Necrotizing fasciitis. Saudi Med J 2001;22:565-568.

5 Elliott D, Kufera JA, Myers RA. The Microbiology of necrotizing soft tissue infections. Am J Surg 2000;179: 361-366.

6 Newton WD, Cramer FS, Norwood SH. Necrotizing fasciitis from invasive Phycomycetes. Crit Care Med 1987;15:331-332.

7 Lakshmi V, Rani TS, Sharma S, et al. Zygomycotic necrotizing fasciitis caused by Apophysomyces elegans. J Clin Microbiol 1993;31:1368-1369.

8 Ribes JA, Vanover-Sams CL, Baker DJ. Zygomycetes in human disease. Clin Microbiol Rev 2000;13: 236-301.

9 Gruhn JG, Sanson J. Mycotic infections in leukemic patients at autopsy. Cancer 1963;16:61-72.

10 Sridhara SR, Paragache G, Panda NK, et al. Mucormycosis in immunocompetent individuals: an increasing trend. J Otolaryngol 2005;34:402-406.

11 Hutter RVP. Phycomycetous infection (mucormycosis) in cancer patients: a complication of therapy. Cancer 1959;12:330-350.

12 Brown AE. Overview of fungal infections in cancer patients. Semin Oncol 1990;17:2-5.

13 Frater JL, Hall GS, Procop GW. Histologic features of zygomycosis: emphasis on perineural invasion and fungal morphology. Arch Pathol Lab Med 2001;125: 375-378.

14 Waldorf AR, Halde C, Vedros NA. Murine model of pulmonary mucormycosis in cortisone-treated mice. Sabouraudia 1982;20:217-224.

15 Spellberg B, Edwards Jr J, Ibrahim A. Novel perspectives on mucormycosis: pathophysiology, presentation, and management. Clin Microbiol Rev 2005;18: $556-569$.

16 Naguib MT, Huycke MM, Pederson JA, et al. Apophysomyces elegans infection in a renal transplant recipient. Am J Kidney Dis 1995;26:381-384.

17 Mathews MS, Raman A, Nair A. Nosocomial zygomycotic post-surgical necrotizing fasciitis in a healthy adult caused by Apophysomyces elegans in South India. J Med Vet Mycol 1997;35: 61-63.

18 Misra PC, Srivastava KJ, Lata K. Apophysomyces, a new genus of the Mucorales. Mycotaxon 1979;8: 377-382.

19 Kimura M, Smith MB, McGinnis MR. Zygomycosis due to Apophysomyces elegans: report of 2 cases and review of the literature. Arch Pathol Lab Med 1999;123:386-390.
20 Chakrabarti A, Panda N, Varma SC, et al. Craniofacial zygomycosis caused by Apophysomyces elegans. Mycoses 1997;40:419-421.

21 Caceres AM, Sardinas C, Marcano C, et al. Apophysomyces elegans limb infection with a favorable outcome: case report and review. Clin Infect Dis 1997;25:331-332.

22 Brown SR, Shah IA, Grinstead M. Rhinocerebral mucormycosis caused by Apophysomyces elegans. Am J Rhinol 1998;12:289-292.

23 Burrell SR, Ostlie DJ, Saubolle M, et al. Apophysomyces elegans infection associated with cactus spine injury in an immunocompetent pediatric patient. Pediatr Infect Dis J 1998;17:663-664.

24 Fairley C, Sullivan TJ, Bartley P, et al. Survival after rhino-orbital-cerebral mucormycosis in an immunocompetent patient. Ophthalmology 2000;107:555-558.

25 Garcia-Covarrubias L, Bartlett R, Barratt DM, et al. Rhino-orbitocerebral mucormycosis attributable to Apophysomyces elegans in an immunocompetent individual: case report and review of the literature. J Trauma 2001;50:353-357.

26 Blair JE, Fredrikson LJ, Pockaj BA, et al. Locally invasive cutaneous Apophysomyces elegans infection acquired from snapdragon patch test. Mayo Clin Proc 2002;77:717-720.

27 Lesueur BW, Warschaw K, Fredrikson L. Necrotizing cellulitis caused by Apophysomyces elegans at a patch test site. Am J Contact Dermat 2002;13:140-142.

28 Wang J, Harvey CM, Calhoun JH, et al. Systemic Apophysomyces elegans after trauma: case report and literature review. Surg Infect (Larchmt) 2002;3: 283-289.

29 Chakrabarti A, Ghosh A, Prasad GS, et al. Apophysomyces elegans: an emerging zygomycete in India. J Clin Microbiol 2003;41:783-788.

30 Carter JE, Ulusarac O. Widespread cutaneous involvement by invasive Apophysomyces elegans in a gravid patient following trauma. Cutis 2003;72:221-228.

31 Kordy FN, Al-Mohsen IZ, Hashem F, et al. Successful treatment of a child with posttraumatic necrotizing fasciitis caused by Apophysomyces elegans: case report and review of literature. Pediatr Infect Dis J 2004;23:877-879.

32 Ruiz CE, Arango M, Correa AL, et al. Necrotizing fasciitis in an immunocompetent patient caused by Apophysomyces elegans. Biomedica 2004;24:239-251.

33 Andresen D, Donaldson A, Choo L, et al. Multifocal cutaneous mucormycosis complicating polymicrobial wound infections in a tsunami survivor from Sri Lanka. Lancet 2005;365:876-878.

34 Patino JF, Castro D, Valencia A, et al. Necrotizing soft tissue lesions after a volcanic cataclysm. World J Surg 1991;15:240-247. 